\title{
The complexity of patellofemoral instability
}

\author{
Roland Becker $^{1} \cdot$ Michael T. Hirschmann ${ }^{2}$. Jon Karlsson ${ }^{3}$
}

Published online: 13 February 2018

(c) European Society of Sports Traumatology, Knee Surgery, Arthroscopy (ESSKA) 2018

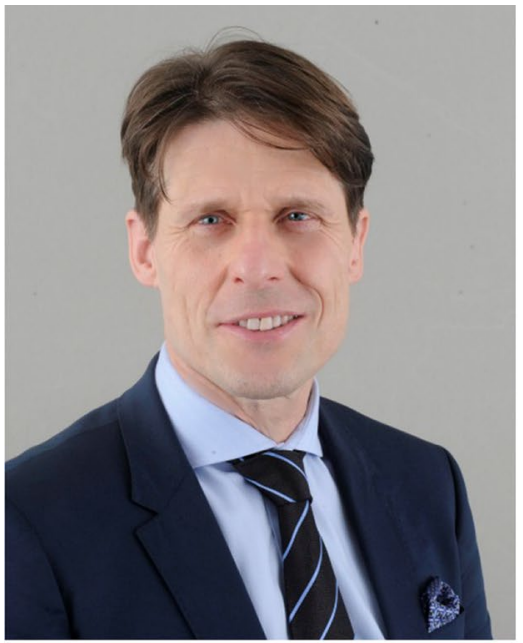

Roland Becker

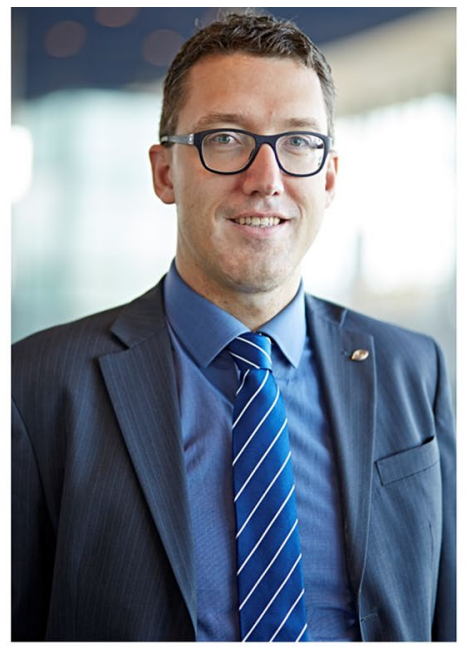

Michael T. Hirschmann

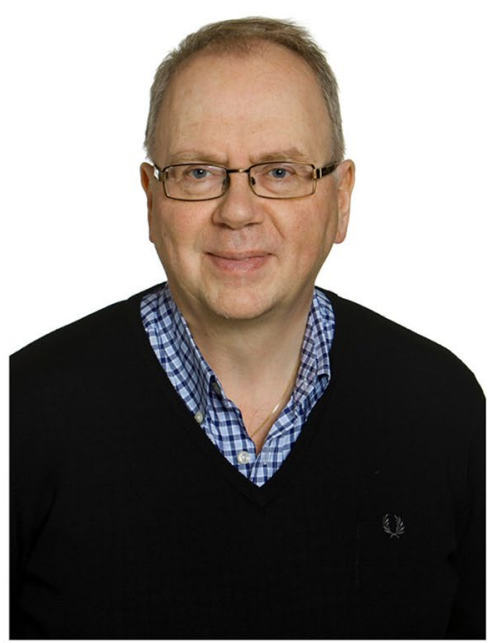

Jon karlsson
The understanding of patellofemoral instability has significantly improved over the last two decades. Patellofemoral pathologies are recognised during every stage of the patient's life. Younger patients typically suffer from dysplasia and patellar instability. Traumatic patellar dislocation generally occurs in young patients and might be of atraumatic or

Roland Becker

roland_becker@yahoo.de

Michael T. Hirschmann

michael.hirschmann@unibas.ch

Jon Karlsson

jon.karlsson@telia.com

1 Department of Orthopaedic and Traumatology, Brandenburg Medical School Theodor Fontane, Hochstrasse 29,

14770 Brandenburg/havel, Germany

2 Department of Orthopaedic Surgery and Traumatology, Kantonsspital Baselland (Bruderholz, Liestal, Laufen), Bruderholz, Switzerland

3 Department of Orthopaedics, Sahlgrenska University Hospital, Sahlgrenska Academy, Gothenburg University, Gothenburg, Sweden traumatic origin. With increasing age patellofemoral osteoarthritis becomes the predominant pathology. Patellofemoral problems might even remain after joint replacement. The patellofemoral compartment causes problems in approximately $25 \%$ of the patients who are not satisfied with the outcome after total knee replacement.

In the October 2014 issue of the KSSTA journal an update was given about the latest clinical and basic science work dealing with the patellofemoral joint. The article by Petersen et al. in that issue is currently the most cited paper of the journal, which highlights the great interest of our readers in patellofemoral topics [6].

Patellar dislocation often occurs in patients with patellar instability which might be caused by a complex variety of different pathologies. The bony alignment, the ligaments and capsule and the function of the quadriceps muscle needs to be evaluated in detail.

The current issue covers several topics, and the three leading papers are dealing predominantly with patellofemoral instability. First at all, risk factors are analysed and presented by the group of Elisabeth Arendt studying 157 patients with first-time patellar dislocation [8]. Special attention is paid 
to the medial patellotibial (MPTL), medial patellomeniscal (MPML) and medial patellofemoral ligaments (MPFL). The MPTL and MPML are secondary restraints to lateral patellar displacement. A detailed review is given about the anatomy, biomechanics and repair techniques of the MPTL and MPML [4]. The amount of being a biomechanical secondary medial stabiliser varies in the literature significantly.

The MPFL, in contrast, is a well-described structure and functions as the main passive stabiliser for the patella near extension. It provides approximately $50 \%$ of the restraint of the medial soft tissues to prevent lateral displacement [1]. Interestingly, the MPFL tears either partially or completely in $87 \%$ of patients after acute patellar dislocation [8]. The site of tear shows a wide variation and was only in $10 \%$ at the femoral and in $17 \%$ at the tibial insertion. The majority of patients presented with multiple rupture locations. This in turn may explain the high failure rate after primary repair on one of the insertion sites.

The semitendinosus tendon has become the most popular graft for reconstruction of the MPFL. However, patellar dislocation requires a complex and detailed diagnostic algorithm to identify the cause of patellofemoral instability, especially when no major trauma is identified. Diagnostic evaluation should include MRI for cartilage assessment and CT scan for assessing bony morphology of the joint. This is important as significant differences have been reported in terms of variation in surface geometry [7]. The tibial tubercle-trochlea groove distance and the rotation of femur and tibia have a significant impact on patellar tracking as well. The femoral rotation needs to be accurately assessed, as the patella should not be forced into the groove, but the groove should be positioned correctly under the patella. In some patients it might require a derotational osteotomy of the femur [2]. All the diagnostic information is needed to decide where and what should be done for stabilising the patella. Only MPFL repair will often not guarantee a stable joint. Soft tissue procedures might fail when patellar tracking is not normalised. Tibial tubercle transfer was traditionally one of the most common procedures for treatment of patellofemoral instability, but only distal malalignment is corrected and combined procedures are often needed. However, in some patients correction osteotomy of the tibial tubercle is required, especially when the tibial tubercle-trochlea grove distance (TT-TG) exceeds $20 \mathrm{~mm}$. The clinical value of a cut-off threshold for the TT-TG distance should be questioned, mainly because the size of knees differs significantly. Thus, the absolute distance of $20 \mathrm{~mm}$ might be inappropriate. For that reason, the surgeon should instead normalise the measured TT-TG distance to the size of the knee [5]. Another study published in the current issue investigated the three lateral quadriceps vectors, which cause patellar subluxation or dislocation measured by the TT-TG distance, Q-angle and tibial sulcus angle (TSA) [3]. A significant correlation was found between these three measurements. The authors concluded that both the TT-TG and the TSA should routinely be taken into consideration to avoid overcorrection.

Patellofemoral instability represents a complex pathology with a considerable number of different influencing factors. For that reason, a detailed understanding of anatomy and kinematics is essential to identify the underlying pathologies. Combined procedures addressing the bone and soft tissues are often necessary. When stabilising the patella it is essential to achieve a correct the patellofemoral tracking with natural mobility. Over-constraining the patella should be avoided under all circumstances, as this is an important factor for the development of osteoarthritis in the medium to long term.

\section{References}

1. Conlan T, Garth WP, Lemons JE (1993) Evaluation of the medial soft-tissue restraints of the extensor mechanism of the knee. $\mathrm{J}$ Bone Jt Surg Am 75:682-693

2. Dickschas J, Harrer J, Pfefferkorn R, Strecker W (2012) Operative treatment of patellofemoral maltracking with torsional osteotomy. Arch Orthop Trauma Surg 132:289-298

3. Graf KH, Tompkins MA, Agel J, Arendt EA (2017) Q-vector measurements: physical examination versus magnetic resonance imaging measurements and their relationship with tibial tubercletrochlear groove distance. Knee Surg Sports Traumatol Arthrosc. https://doi.org/10.1007/s00167-017-4527-5

4. Hinckel BB, Gobbi RG, Kaleka CC, Camanho GL, Arendt EA (2017) Medial patellotibial ligament and medial patellomeniscal ligament: anatomy, imaging, biomechanics, and clinical review. Knee Surg Sports Traumatol Arthrosc. https://doi.org/10.1007/ s00167-017-4469-y

5. Hingelbaum S, Best R, Huth J, Wagner D, Bauer G, Mauch F (2014) The TT-TG index: a new knee size adjusted measure method to determine the TT-TG distance. Knee Surg Sports Traumatol Arthrosc 22:2388-2395

6. Petersen W, Ellermann A, Gösele-Koppenburg A, Best R, Rembitzki IV, Brüggemann GP, Liebau C (2014) Patellofemoral pain syndrome. Knee Surg Sports Traumatol Arthrosc 22:2264-2277

7. Tecklenburg K, Dejour D, Hoser C, Fink C (2006) Bony and cartilaginous anatomy of the patellofemoral joint. Knee Surg Sports Traumatol Arthrosc 14:235-240

8. Tompkins MA, Rohr SR, Agel J, Arendt EA (2017) Anatomic patellar instability risk factors in primary lateral patellar dislocations do not predict injury patterns: an MRI-based study. Knee Surg Sports Traumatol Arthrosc. https://doi.org/10.1007/s0016 7-017-4464-3 